\title{
Dietary Patterns and Mild Cognitive Impairment Risk in Korean Adults over 50 Years Old
}

\author{
Kyoung Yun $\mathrm{Kim}^{1}$ and Jung-Mi Yun ${ }^{2}$ \\ ${ }^{1}$ Sun-Han Hospital, Gwangju 61917, Korea \\ ${ }^{2}$ Department of Food and Nutrition, Chonnam National University, Gwangju 61186, Korea
}

\begin{abstract}
The prevalence of age-related diseases such as dementia and cognitive disorders is rapidly increasing. This study aimed to identify the dietary patterns associated with mild cognitive impairment (MCI) in adults aged over 50 years. This cross-sectional study investigated dietary patterns associated with cognitive function among older adults hospitalized in Gwangju province. Global cognitive function was assessed using the Mini-Mental State Examination. Diet information was obtained using a food frequency questionnaire with 112 food items and 24-h dietary recall. Using a principal component analysis, we identified three dietary patterns, "legumes and vegetables", "beverage and nuts", and "white rice". The "beverage and nuts" pattern was inversely associated with the prevalence of high $\mathrm{MCI}$ after adjusting for covariates (third vs. first tertile, adjusted odds ratio: $0.333 ; 95 \%$ confidence interval: $0.133 \sim 0.831 ; P<0.05$ ). The white rice pattern was associated with the prevalence of $\mathrm{MCI}$ in the crude analysis. However, after adjusting for all confounding factors, no association was found. The "beverage and nuts" pattern was inversely associated with the prevalence of MCI. In the future, longitudinal population-based studies and randomized clinical trials are required to confirm the effect of potential dietary patterns on cognitive impairment and reveal the underlying mechanism of their association.
\end{abstract}

Keywords: diet, mild cognitive impairment, principal component analysis

\section{INTRODUCTION}

The prevalence of age-related diseases, such as dementia and cognitive impairment, is rapidly increasing worldwide. In 2013, the World Health Organization reported that there were about 47.5 million patients with dementia globally in 2010, with 7.7 million patients newly diagnosed each year (Wimo et al., 2013). As a result, the cost of dementia worldwide is estimated to be 604 billion dollars per year (Wimo et al., 2013). In a study of 9,485 Koreans conducted in 2010 and 2011, the prevalence of dementia and mild cognitive impairment (MCI) in participants aged over 65 years was $5.4 \%$ and $4.3 \%$, respectively (Jang et al., 2014). MCI is considered to be the transitional state between the expected cognitive decline of normal aging and dementia progression (Albert and Blacker, 2006). Although people with $\mathrm{MCI}$ have a greater risk of dementia, many studies have reported that it is possible to prevent progression to dementia by controlling environmental factors such as dietary habits, exercise, and chronic disease management (Eshkoor et al., 2015; Jiang et al.,
2017). Several studies have recently investigated the associations between cognitive function and dietary factors, including certain foods and nutrients (Panza et al., 2015; Jiang et al., 2017). Adequate consumption of omega-3 fatty acids (Cederholm et al., 2013), fruits and vegetables (Dong et al., 2016; Jiang et al., 2017), dairy products (Ogata et al., 2016), and moderate alcohol consumption (Xu et al., 2017) have been reported to have protective effects against disease. However, the association between dietary factors and the risk of cognitive impairment and dementia remains unclear (Panza et al., 2015; KesseGuyot et al., 2016; Smith and Blumenthal, 2016).

Recently, dietary patterns have been used to examine how diseases can be prevented and disease conditions improved (Ozawa et al., 2013; Kim et al., 2015; van de Rest et al., 2015; Shin et al., 2018). As we consume a number of different types of food, rather than a single type of food, it seems reasonable to investigate the effects of dietary patterns on disease. Thus, research using dietary patterns, considering the interaction and synergy of nutrients in foods, is valid for disease prevention studies 
(Hoffmann et al., 2004; Ozawa et al., 2013; Kim et al., 2015). Accelerated economic development and globalization have changed traditional Korean dietary patterns. This change has resulted in Koreans consuming fewer food crops, such as rice, but increased amounts of bread, meat, and seafood (Lee and Cho, 2014). Thus, over time, the diet of Koreans has become more westernized. Western dietary habits are known to affect the incidence of and mortality due to chronic diseases such as metabolic syndrome and cardiovascular disease (Lee and Cho, 2014). Thus, it is necessary to investigate the current daily dietary patterns of Koreans.

Factor analysis, cluster analysis, reduced rank regression, and partial least-squares regression have been used to identify dietary patterns. A factor analysis is a statistical technique that considers the correlation between variables, reduces the order of variables through common underlying dimensions, and generally uses a principal component analysis (PCA) (Hoffmann et al., 2004).

This study aimed to identify the dietary patterns of elderly Koreans with MCI using a PCA and determine their impact on cognitive function so as to contribute to the provision of healthy dietary guidelines.

\section{MATERIALS AND METHODS}

\section{Study design and participants}

Data of 324 older adults aged over 50 years hospitalized in Gwangju Sun-Han Hospital were collected through a face-to-face interview with questionnaires from July 2017 to March 2018. We excluded participants with very high or low total energy intake levels ( $<500$ or $>3,500 \mathrm{kcal}$ ), those who were on diet therapy within the last year (as this would change their daily dietary patterns), or those with a severe mental disorder, metabolic diseases, cancer, alcohol abuse, Parkinson's disease, and/or Alzheimer's disease. Two hundred and seventy-five participants, including 104 males and 171 females, completed all of the questionnaires, including a Mini-Mental State Examination (MMSE), short-form geriatric depression scales, 24-h dietary recall, and a semi-quantitative food frequency questionnaire (SQ-FFQ). This study complied with the tenets of the Declaration of Helsinki, and all procedures involving human participants were approved by the Institutional Review Board of Chonnam National University (1040198-180731-HR-071-01). All participants who participated in our study signed a consent form.

\section{Dietary assessment}

Data were obtained by trained dietitians who interviewed all the participants face-to-face. If necessary, patients' caregivers helped with the completion of the dietary intake survey.
A food and nutrient intake survey was conducted using only the 24-h dietary recall method considering the age of the participants and SQ-FFQ with 112 food items. The FFQ examines the food intake of participants, assuming that their dietary habits do not change frequently. The 24-h dietary recall method provides more accurate information on the food that participants have consumed. It has been reported that using the two methods in parallel makes it possible to capture dietary habits more accurately (Freedman et al., 2018). The SQ-FFQ has been reported to be valid and reproducible previously (Feskanich et al., 1993). Participants were asked to check each of the nine frequency ranges from "none" to "three times a day" for food and beverages. Daily food intake derived from the FFQ was calculated by multiplying the food intake frequency of each standard serving size. The daily nutrient intake was calculated by multiplying the intake frequency and standard portion size according to CAN-PRO version 4.0 (Computer-Aided Nutritional Analysis Program, the Korea Nutrition Society, Seoul, Korea).

\section{Cognitive function assessment}

We used the MMSE tool, a global test (Huang et al., 2009), to measure cognitive function. The MMSE includes 30 items covering the following fields: time and place orientation, memory registration and recall, attention and calculation, language function, and understanding and judgment. Scores were corrected according to education level and ranged from 0 to 30. Higher MMSE scores indicate better cognitive function. Participants were assigned to the MCI group if their MMSE score was $19 \sim 24$ and to the normal group if their MMSE score was $25 \sim 30$, according to the MCI clinical diagnosis cut-off value mentioned in a previous study (Huang et al., 2009). The sensitivity and accuracy of a score below 24 on the MMSE, defined as cognitive impairment, is $80 \sim 90 \%$ and $80 \sim 100 \%$, respectively (Tombaugh and McIntyre, 1992). Finally, the Sun-Han medical staff confirmed the diagnosis of the participants with MCI. The short geriatric depression scale (SGDS) developed in 1986 is a tool used for screening older adults for symptoms of depression (Greenberg, 2007). This self-reported screening tool consists of 15 questions that can be completed quickly using "yes" or "no" answers, making it useful in the community setting. A score of $0 \sim 4$ is not typically a cause for concern, $5 \sim 8$ suggests mild depression, 9 11 suggests moderate depression, and 12 15 suggests severe depression (Greenberg, 2007). The SGDS tool has been reported to identify $92 \%$ of people with depression (Sheikh and Yesavage, 1986).

\section{PCA and identification of dietary patterns}

Dietary patterns were generated by utilizing the PCA for 21 predefined food groups (Khosravi et al., 2015; Kim et 
al., 2015; Table 1). In this study, the food items were combined into food groups based on their nutrient contents and uses. Besides, we divided the vegetable group into salty vegetables and vegetables to consider whether the salt difference had any impact.

A principal component and factor analysis is a nutritional epidemiology method that derives dietary patterns by distinguishing one or more factors based on foods that tend to (or are not) ingested by the same subject (Osler et al., 2002; Schulze et al., 2003). The PCA explains the frequency of various foods or food groups consumed by the individual as a linear function of the principal components. That is, the first principal component accounts for the maximum amount of variation among individuals. The second principal component is derived from the orthogonal rotation of the first principal component and accounts for the maximum amount of variance among the factors. Finally, the PCA identifies dietary patterns by analyzing the intake frequency based on the correlation matrix of the foods included in the survey (Schulze et al., 2003).

To extract the dietary patterns, we classified 112 food items into 21 food groups with reference to similar nutrient profiles and previous studies: white rice, multigrain rice, flour-based food, rice-cakes, bread, soup and stew, legumes, eggs, red meat and processed meat, poultry, fish, vegetables, salty vegetables, seaweed, fruit, dairy products, coffee and tea, beverages, nuts, snacks, and alcohol. The number of dietary patterns (referred to as derived factors) was determined using an eigenvalue of $>1.25$ and scree plot (Schulze et al., 2003). Furthermore, the factors were rotated with an orthogonal transformation

Table 1. Food grouping used in the dietary intake analysis

\begin{tabular}{|c|c|}
\hline Food groups & Food items \\
\hline White rice & Cooked white rice, fried rice, cooked rice with assorted mixtures, rice rolled in laver, curry and rice, cereal \\
\hline Multigrain rice & Cooked rice with other grains and legumes \\
\hline Flour-based foods & $\begin{array}{l}\text { Instant noodles, instant cup noodles, noodles, kalguksu, udong, Chinese black bean noodles, spicy seafood } \\
\text { noodle soup, cold noodles, dumpling (steamed or fried) }\end{array}$ \\
\hline Rice-cake & $\begin{array}{l}\text { Plain steamed rice-cake, steamed rice-cake with red bean, cubed rice-cake with soybean powder, plain } \\
\text { cubed rice cake, seasoned bar rice-cake }\end{array}$ \\
\hline Bread & $\begin{array}{l}\text { Loaf bread, sweet red-beans buns, steamed sweet red-bean buns, cream buns, sponge cake (castella), cake, } \\
\text { chocopie }\end{array}$ \\
\hline Soup and stew & $\begin{array}{l}\text { Rice-cake soup, beef born and meat potage, potato and pork rib soup, loach stew, frozen Alaska pollack } \\
\text { stew, spicy seafood stew, sea mustard soup, dried Alaska pollack soup, beef soup, spicy beef soup, radish } \\
\text { soup, bean paste soup, bean paste stew, fermented soybean stew, kimchi stew, stir-fried kimchi, spicy } \\
\text { sausage stew, bean curd stew, soft bean curd stew }\end{array}$ \\
\hline Legume & Bean curd, bean curd boiled in soy sauce, pan-fried bean curd, soybean boiled in soy sauce \\
\hline Eggs & Fried egg, fried egg roll, boiled egg, steamed egg \\
\hline $\begin{array}{l}\text { Red meats and } \\
\text { processed meats }\end{array}$ & $\begin{array}{l}\text { Pizza, hamburger, sandwich, grilled pork belly, boiled pork, stir-fried pork (sweet, spicy), grilled pork ribs, } \\
\text { steamed pork ribs, grilled beef, stir-fried beef, sweet and sour pork, pork cutlet, ham, pork roll }\end{array}$ \\
\hline Poultry & Korean traditional chicken soup, stir-fried chicken, chicken boiled with soy sauce, fried chicken, grilled duck \\
\hline Fish & $\begin{array}{l}\text { Mackerel, saury (grill, boiled with soy sauce), hairtail, croaker (grill, boiled with soy sauce), anchovy, stir-fried } \\
\text { anchovy, squid (raw, boiled, stir-fried), dried shredded squid (stir-fried, seasoned), dried squid, crab } \\
\text { preserved in soy or spicy sauce, salted shrimp, squid and clam, fish ball (stir-fried, soup) }\end{array}$ \\
\hline Vegetables & $\begin{array}{l}\text { Stir-fried potatoes, potatoes boiled with soy sauce, steamed potatoes, grilled potatoes, steamed sweet } \\
\text { potatoes, grilled sweet potatoes, steamed corn, grilled corn, bean sprout (seasoned, soup), seasoned mung } \\
\text { bean sprout, seasoned spinach, seasoned bellflower (boiled or not), pumpkin (seasoned, pan-fried), } \\
\text { seasoned other vegetables, cucumber (seasoned, raw), radish (seasoned, pickled, dried), vegetables salad, } \\
\text { seasoned green onion, seasoned Chinese chive, raw vegetables (lettuce, sesame, Chinese cabbage, } \\
\text { pumpkin leaf), green pepper, boiled broccoli, boiled cabbage, garlic, lotus roots boiled with soy sauce, } \\
\text { burdock boiled with soy sauce, Korean pancake (Chinese chive pancake, kimchi pancake), stir-fried } \\
\text { vegetable and noodles, stir-fried mushroom, soybean paste sauce }\end{array}$ \\
\hline Salty vegetables & Korean cabbage kimchi, other kimchi, pickle \\
\hline Seaweeds & $\begin{array}{l}\text { Grilled laver, raw laver, seasoned laver, seasoned green laver, seasoned brown seaweed, stir-fried sea } \\
\text { mustard stems }\end{array}$ \\
\hline Fruits & $\begin{array}{l}\text { Strawberry, tomato, cherry tomato, melon, water melon, peach, grape, apple, pear, persimmon, dried } \\
\text { persimmon, tangerine, banana, orange, kiwi }\end{array}$ \\
\hline Dairy products & Milk (low fat, normal), liquid type yogurt, curd type yogurt, soybean milk \\
\hline Coffee and tea & Coffee, green tea \\
\hline Beverages & Soft drink (cola, soda, fruit juice soda), fruit juice, grain powder beverage, rice beverage \\
\hline Nuts & Peanut, chestnut \\
\hline Snack & Snack, cookie, cracker, chocolate, ice cream, ices \\
\hline Alcohol & Soju, beer, rice wine \\
\hline
\end{tabular}

This study reorganized the foods containing similar nutrients into new groups based on previous studies (Khosravi et al., 2015; Kim et al., 2015). 
using a varimax rotation to achieve a simpler structure with easier interpretability (Kline, 1994). We considered the food groups with an absolute factor loading $>0.2$ to be significant in the calculation of pattern scores because the food items included in these food groups appeared to have a strong association with the identified factors (Kline, 1994; McCann et al., 2001). The factor scores were calculated by summing each subject's intake of the 21 food groups weighted by the factor loadings. Each dietary pattern score was categorized by tertile, with a higher tertile indicating better adherence. We labeled the dietary pattern according to the food with the highest factor loadings.

\section{Energy and nutrient intake using the 24-h recall method} Nutrient intakes were estimated by multiplying the intake frequency and standardized portion size for each food. The amount of nutrients contained per gram of food was obtained from CAN-PRO version 4.0. Daily nutrient intakes of participants were the sum of their intake of the 112 food items. Macro- and micronutrient intakes were adjusted for in the total energy intake using the residual method. Considering the age of the participants, the dietary survey was performed using ancillary equipment such as a measuring cup, photographs of the prescribed amount of food, and tableware. The nutrient variables were used as continuous data of the daily intake of total energy ( $\mathrm{kcal} / \mathrm{d})$, carbohydrates (g/1,000 kcal), fat (g/1,000 kcal), protein $(\mathrm{g} / 1,000 \mathrm{kcal})$, saturated fatty acid $(\mathrm{g} / 1,000 \mathrm{kcal})$, monounsaturated fatty acid (MUFA; g/1,000 kcal), polyunsaturated fatty acid (PUFA; g/1,000 kcal), fiber (g/ $1,000 \mathrm{kcal})$, water $(\mathrm{g} / 1,000 \mathrm{kcal}), \beta$-carotene $(\mu \mathrm{g}$ retinol equivalent $/ 1,000 \mathrm{kcal})$, vitamin $\mathrm{E}(\mathrm{mg} / 1,000 \mathrm{kcal})$, vitamin $\mathrm{C}(\mathrm{mg} / 1,000 \mathrm{kcal})$, thiamin $(\mathrm{mg} / 1,000 \mathrm{kcal})$, niacin $(\mathrm{mg} / 1,000 \mathrm{kcal})$, vitamin B6 (mg/1,000 kcal), folate $(\mu \mathrm{g} /$ $1,000 \mathrm{kcal})$, vitamin B12 (mg/1,000 kcal), calcium $(\mathrm{mg} /$ $1,000 \mathrm{kcal})$, and cholesterol (mg/1,000 kcal).

\section{Covariates}

We interviewed all the participants face-to-face. The participants were informed that their data would be handled confidentially. The survey was conducted as quickly as possible due to the age of the participants.

Participants' general characteristics such as age, sex, education level, inhabitation, self-measured health status level, prescribed medications, self-reported dental condition level, and sleep duration were collected. Furthermore, the following information was collected: alcohol consumption status (if less than 12 times per year with less than one glass per drink then the participant was classified as no, former, and current drinkers was classified as a yes), smoking status (never was classified as no, former, and current smokers were classified as yes), physical activity in leisure time (no, usually, and yes), break- fast frequency/week, and nutritional supplements. Participants had standardized anthropometric measures taken by trained nurses. Body mass index was calculated as a participant's body weight in kilograms divided by their height in meters squared. A trained nurse measured each participant's blood pressure using an automatic blood pressure monitor (HBP-9020, Omron Healthcare Co., Ltd., Kyoto, Japan) after allowing the participant to rest for $5 \mathrm{~min}$ beforehand. Then, subject's blood pressure was repeatedly measured, with their arms and back in a straight line and their arms in line with their heart.

\section{Statistical analysis}

Categorical variables were expressed as frequency and percentage (\%), and continuous variables as mean \pm standard deviation. We investigated the association between cognitive function and dietary patterns using a logistic regression analysis. Dietary patterns were determined using a factor analysis and PCA. We considered the correlation between the measured variables and extracted the factors by examining the calculated correlation matrix. Factor loading was calculated from the extracted factors. To simplify the column of the factor matrix, varimax rotation was performed.

The effect of each dietary pattern on cognitive function as the tertile increases from $\mathrm{T} 1$ to $\mathrm{T} 3$ was estimated using the odds ratio. The confounding factors of the analysis were as follows: sex, age, inhabitation, education, selfreported dental condition, sleep duration, alcohol consumption status, smoking status, physical activity in leisure time, nutritional supplements, and the SGDS variable to analyze the risk of $\mathrm{MCI}$ with each dietary pattern score (Model 1). A $P$-value of $<0.05$ was considered statistically significant. All statistical analyses were performed using SPSS version 18.0 (IBM Corp., Armonk, NY, USA).

\section{RESULTS}

\section{General and anthropometric characteristics of the participants according to the MMSE score}

According to the MMSE score, the general and anthropometric characteristics of participants were analyzed and are presented in Table 2. Participants with MCI (70.5 years) tended to be older than normal participants (63.8 years). Normal participants were more highly educated than the participants with MCI. Furthermore, $75.5 \%$ of normal participants and $55.7 \%$ of the participants with MCI lived alone. In addition, $32.9 \%$ of participants with MCI responded that their health status was poor. Sleep duration was shorter in participants with $\mathrm{MCI}(4 \sim 6 \mathrm{~h} / \mathrm{d})$ than normal participants $(6 \sim 8 \mathrm{~h} / \mathrm{d})$. Participants who self-reported SGDS $\geq 12$ were more likely to be in the 
Table 2. General characteristics of participants according to MMSE score

\begin{tabular}{|c|c|c|c|c|}
\hline \multirow[b]{2}{*}{ Characteristics } & \multicolumn{3}{|c|}{ MMSE } & \multirow[b]{2}{*}{$P$-value } \\
\hline & $\begin{array}{c}\text { Total } \\
(n=275)\end{array}$ & $\begin{array}{l}19 \sim 24 \\
(n=79)\end{array}$ & $\begin{array}{c}\geq 25 \\
(n=196)\end{array}$ & \\
\hline Sex & & & & 0.606 \\
\hline Men & $104(37.8)$ & $28(35.4)$ & $76(38.8)$ & \\
\hline Women & $171(62.2)$ & $51(64.6)$ & $120(61.2)$ & \\
\hline Age (yrs) & $65.7 \pm 9.4$ & $70.5 \pm 9.5$ & $63.8 \pm 9.2$ & $<0.001$ \\
\hline $50 \sim 64$ & $130(47.3)$ & $19(24.1)$ & $111(56.6)$ & \\
\hline $65 \sim 74$ & 97 (35.3) & $35(44.3)$ & $62(31.6)$ & \\
\hline$\geq 75$ & $48(17.5)$ & $25(31.6)$ & $23(11.7)$ & \\
\hline Education & & & & $<0.001$ \\
\hline Illiterate & $115(41.8)$ & $49(62.0)$ & $66(33.7)$ & \\
\hline Junior high school & $42(15.3)$ & $6(7.6)$ & $36(18.4)$ & \\
\hline High school & $80(29.1)$ & $19(24.1)$ & $61(31.1)$ & \\
\hline Above & $38(13.8)$ & $5(6.3)$ & $33(16.8)$ & \\
\hline Inhabitation & & & & 0.001 \\
\hline Alone & $192(69.8)$ & $44(55.7)$ & $148(75.5)$ & \\
\hline With spouse & $83(30.2)$ & $35(44.3)$ & $48(24.5)$ & \\
\hline Self-reported health status ${ }^{1)}$ & & & & 0.024 \\
\hline Poor & $64(23.8)$ & $26(32.9)$ & $38(20.0)$ & \\
\hline Good or fair & $205(76.2)$ & $53(67.1)$ & $152(80.0)$ & \\
\hline Medication (yes) & $205(74.5)$ & $68(86.1)$ & $137(69.9)$ & 0.005 \\
\hline Current disease (yes) & $195(70.9)$ & $62(78.5)$ & $133(67.9)$ & 0.079 \\
\hline Self-reported dental condition & & & & 0.145 \\
\hline Very good or good & $184(66.9)$ & $58(73.4)$ & $126(64.3)$ & \\
\hline Very poor or poor & $91(33.1)$ & $21(26.6)$ & $70(35.7)$ & \\
\hline Sleep duration $(\mathrm{h} / \mathrm{d})$ & & & & 0.002 \\
\hline$<4$ & $17(6.2)$ & $11(13.9)$ & $6(3.1)$ & \\
\hline $4 \sim 6$ & $126(45.8)$ & $40(50.6)$ & $86(43.9)$ & \\
\hline $6 \sim 8$ & $121(44.0)$ & $26(32.9)$ & $95(48.5)$ & \\
\hline$\geq 8$ & $11(4.0)$ & $2(2.5)$ & $9(4.6)$ & \\
\hline Alcohol consumption (yes) & $107(38.9)$ & $23(29.1)$ & $86(43.9)$ & 0.024 \\
\hline Smoking (yes) & $213(77.5)$ & $66(83.5)$ & $147(75.0)$ & 0.125 \\
\hline Physical activity in leisure time & & & & 0.083 \\
\hline No & $131(47.6)$ & $46(58.2)$ & $85(43.4)$ & \\
\hline Usually & $88(32.0)$ & $20(25.3)$ & $68(34.7)$ & \\
\hline Yes & $56(20.4)$ & $13(16.5)$ & $43(21.9)$ & \\
\hline Breakfast frequency (weekly, times) & & & & 0.969 \\
\hline $5 \sim 7$ & $257(93.5)$ & $74(93.7)$ & $183(93.4)$ & \\
\hline $3 \sim 4$ & $10(3.6)$ & $3(3.8)$ & $7(3.6)$ & \\
\hline$\leq 2$ & $8(2.9)$ & $2(2.5)$ & $6(3.1)$ & \\
\hline Nutritional supplements (usually or yes) & $229(83.3)$ & $63(79.7)$ & $166(84.7)$ & 0.320 \\
\hline SGDS $^{11}$ & & & & $<0.001$ \\
\hline Normal $(\leq 4)$ & $197(76.4)$ & $32(47.8)$ & $165(86.4)$ & \\
\hline Mild or moderate $(5 \sim 11)$ & $40(15.5)$ & $20(29.9)$ & $20(10.5)$ & \\
\hline Severe $(\geq 12)$ & $21(8.1)$ & $15(22.4)$ & $6(3.1)$ & \\
\hline BMI $\left(\mathrm{kg} / \mathrm{m}^{2}\right)$ & $23.7 \pm 3.8$ & $23.6 \pm 3.6$ & $23.8 \pm 3.9$ & 0.702 \\
\hline $\mathrm{SBP}(\mathrm{mmHg})$ & $117.4 \pm 12.9$ & $120.1 \pm 14.7$ & $116.4 \pm 11.0$ & 0.024 \\
\hline $\mathrm{DBP}(\mathrm{mmHg})$ & $75.8 \pm 11.5$ & $77.8 \pm 12.5$ & $75.0 \pm 10.4$ & 0.056 \\
\hline
\end{tabular}

Values are expressed as the number of participants for each category (\%) or mean \pm standard deviation.

MMSE, mini-mental state examination; SGDS, short geriatric depression scale; BMI, body mass index; SBP, systolic blood pressure; $\mathrm{DBP}$, diastolic blood pressure.

${ }^{1)}$ The response base differs because there are cases of irrelevant responses or no responses.

$P$-values were obtained from chi-square test for categorical variables.

MCI group (22.4\%) than the normal group (3.1\%). Systolic blood pressure was higher in the MCI group (120.1 $\mathrm{mmHg}$ ) than the normal group (116.4 $\mathrm{mmHg})$.

\section{Factor loadings and dietary patterns from the PCA}

Based on the scree plot, we derived three dietary patterns with eigenvalues of $\geq 1.6,46.85 \%$ of the cumulative explained variation among the 21 food groups. Table 3 and 
Table 3. Factor loadings and variation in food groups, and dietary patterns from principal component analysis

\begin{tabular}{|c|c|c|c|}
\hline Food groups & Factor $1^{1)}$ & Factor $2^{2)}$ & Factor $3^{3)}$ \\
\hline Legume & 0.835 & - & - \\
\hline Vegetables & 0.775 & - & - \\
\hline Seaweeds & 0.770 & - & - \\
\hline Soup and stew & 0.711 & - & - \\
\hline Eggs & 0.648 & - & - \\
\hline Fish & 0.623 & - & - \\
\hline Poultry & 0.564 & - & - \\
\hline $\begin{array}{l}\text { Red meats and } \\
\text { processed meats }\end{array}$ & 0.513 & - & - \\
\hline Beverages & - & 0.711 & - \\
\hline Nuts & - & 0.609 & - \\
\hline Snack & - & 0.604 & - \\
\hline Fruits & - & 0.559 & - \\
\hline Rice-cake & - & 0.474 & - \\
\hline Coffee and tea & - & 0.393 & - \\
\hline Bread & - & 0.298 & - \\
\hline Salty vegetables & - & -0.257 & - \\
\hline White rice & - & - & 0.662 \\
\hline Flour-based foods & - & - & 0.629 \\
\hline Alcohol & - & - & 0.563 \\
\hline Dairy products & - & - & -0.485 \\
\hline Multigrain rice & - & - & -0.430 \\
\hline Eigenvalue & 6.137 & 2.041 & 1.661 \\
\hline $\begin{array}{l}\text { Cumulative } \\
\text { explained variation }\end{array}$ & 21.347 & 35.302 & 46.854 \\
\hline
\end{tabular}

Kaiser-Meyer-Olkin=0.821, Bartlett's test results=2,270.582, and $\mathrm{df}=210$, and Sig=0.000.

The following factors had loadings $\geq|0.20|$ are shown in the table. The score for each dietary pattern was estimated from the 21 predefined food groups.

Legume and vegetables pattern include legume, vegetables, seaweeds, soup and stew, eggs, fish, poultry, red meats and processed meats.

1)“Legume and vegetables” pattern was positively characterized by high consumption of legume, vegetables, seaweeds, soup and stew, eggs, fish, poultry, and red meats and processed meat.

2)“Beverage and nuts" pattern was positively characterized by high consumption of beverages, nuts, sweet foods, fruits, rice-cake, coffee and tea, and bread.

3) "White rice" pattern was characterized by higher consumption of white rice, flour-based foods and alcohol and lower consumption of dairy products, and multigrain rice.

Fig. 1 illustrate the factor loadings $(\geq|0.20|)$, which characterize each dietary pattern. The first dietary pattern, "legumes and vegetables", was positively characterized by a high consumption of legumes, vegetables, seaweed, soup and stew, eggs, fish, poultry, red meat, and processed meat (Fig. 1). Furthermore, the second dietary pattern (mix of healthy and unhealthy food groups), "beverage and nuts", was positively characterized by a high consumption of beverages, nuts, sweet foods, fruit, ricecakes, coffee and tea, and bread (Fig. 1). The "white rice" pattern was characterized by a high consumption of white rice, flour-based food, and alcohol, and a low consumption of dairy products and multigrain rice (Fig. 1).

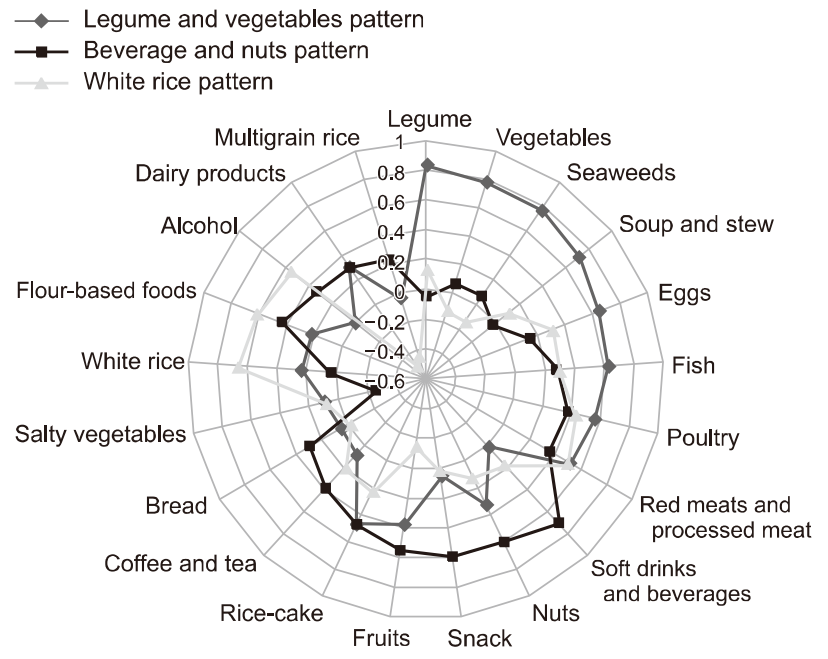

Fig. 1. Radar graph of factor loadings characterizing 3 dietary patterns. Factor scores were calculated by summing the 21 food groups' intake frequency weighted by the factor loading.

\section{Characteristics of participants with different dietary patterns}

Table 4 and Fig. 2 presents the characteristics of participants according to the derived dietary pattern score tertile. The level of education and physical activity increased across the tertiles of the "beverage and nuts" pattern. However, the self-perceived dental condition was very poor or poor in higher tertiles in this dietary pattern. Selfreported depressive symptom scores were lower in T3 (score: 3.4 ) than in $\mathrm{Tl}$ (score: 4.6), and participants with severe status (score: $\geq 12$ ) were less prevalent in T3 (7.4\%) than T1 (18.1\%). The MMSE score was higher in higher tertiles (Tl score: 26.0 vs. T3 score: 28.1 ). As the tertile increased in the "white rice" pattern, the distribution of males and participants $\geq 75$ years was augmented. The proportion of participants who slept $<4$ $\mathrm{h} / \mathrm{d}$ increased from T1 to T3. The proportion of former and current smokers was higher in T1 (82.4\%). As the tertile increased, the self-reported SGDS score (Tl score: 2.6 vs. T3 score: 4.5 ) increased, and the MMSE score (Tl score: 28.0 vs. T3 score: 25.9 ) decreased. The anthropometric characteristics of participants were not significant in all patterns.

\section{Energy and nutrient intake level according to the dietary pattern}

Energy and nutrient intakes according to each dietary pattern score tertile are presented in Table 5. The nutrient intake of the legumes and vegetable pattern did not show any significant difference according to the tertile. $\beta$-Carotene, vitamin $C$, and folate significantly increased as the "beverage and nuts" pattern score increased from $\mathrm{T} 1$ to T3 $(P<0.05)$. There was no significant difference in other nutrients by tertile in the white rice pattern except for thiamin. 


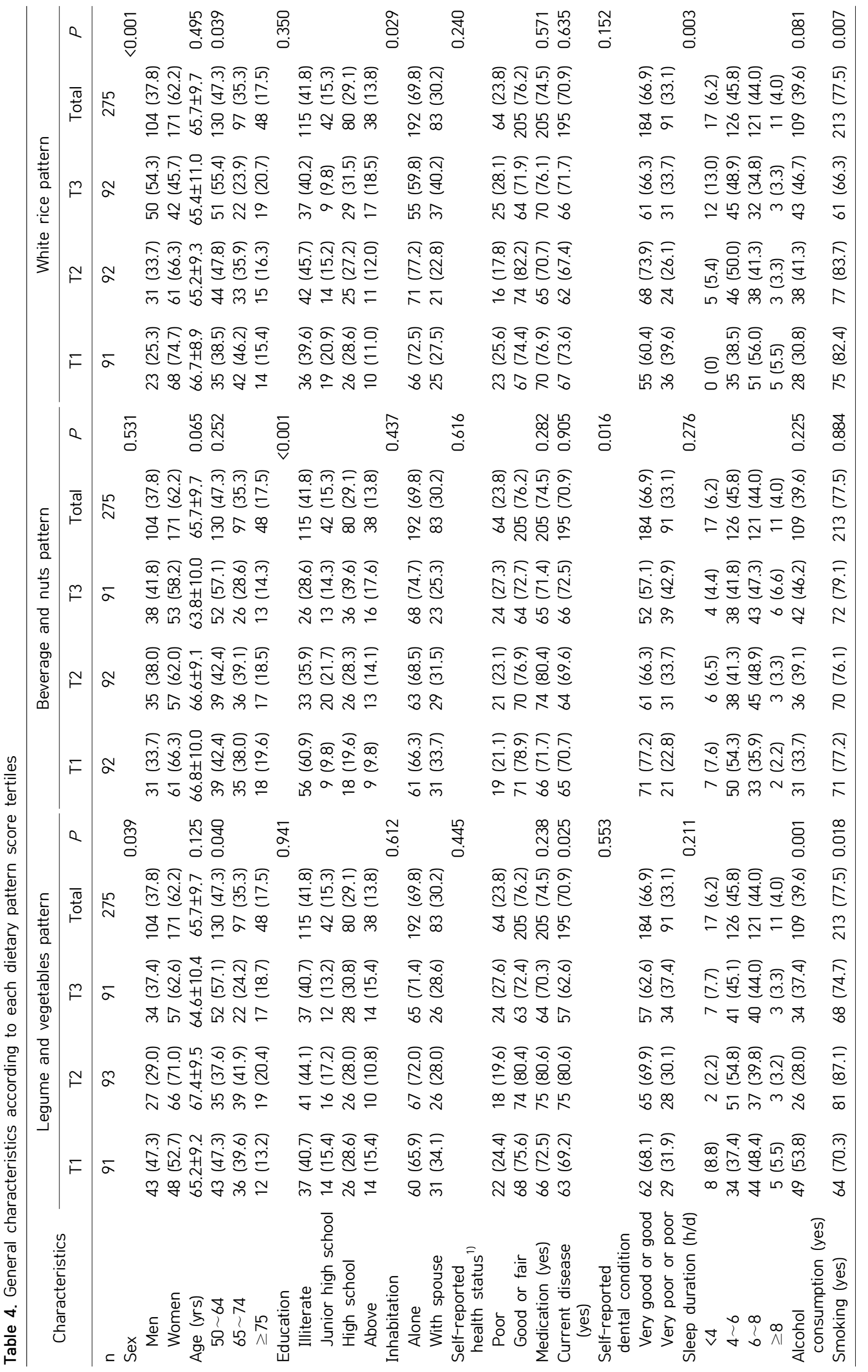




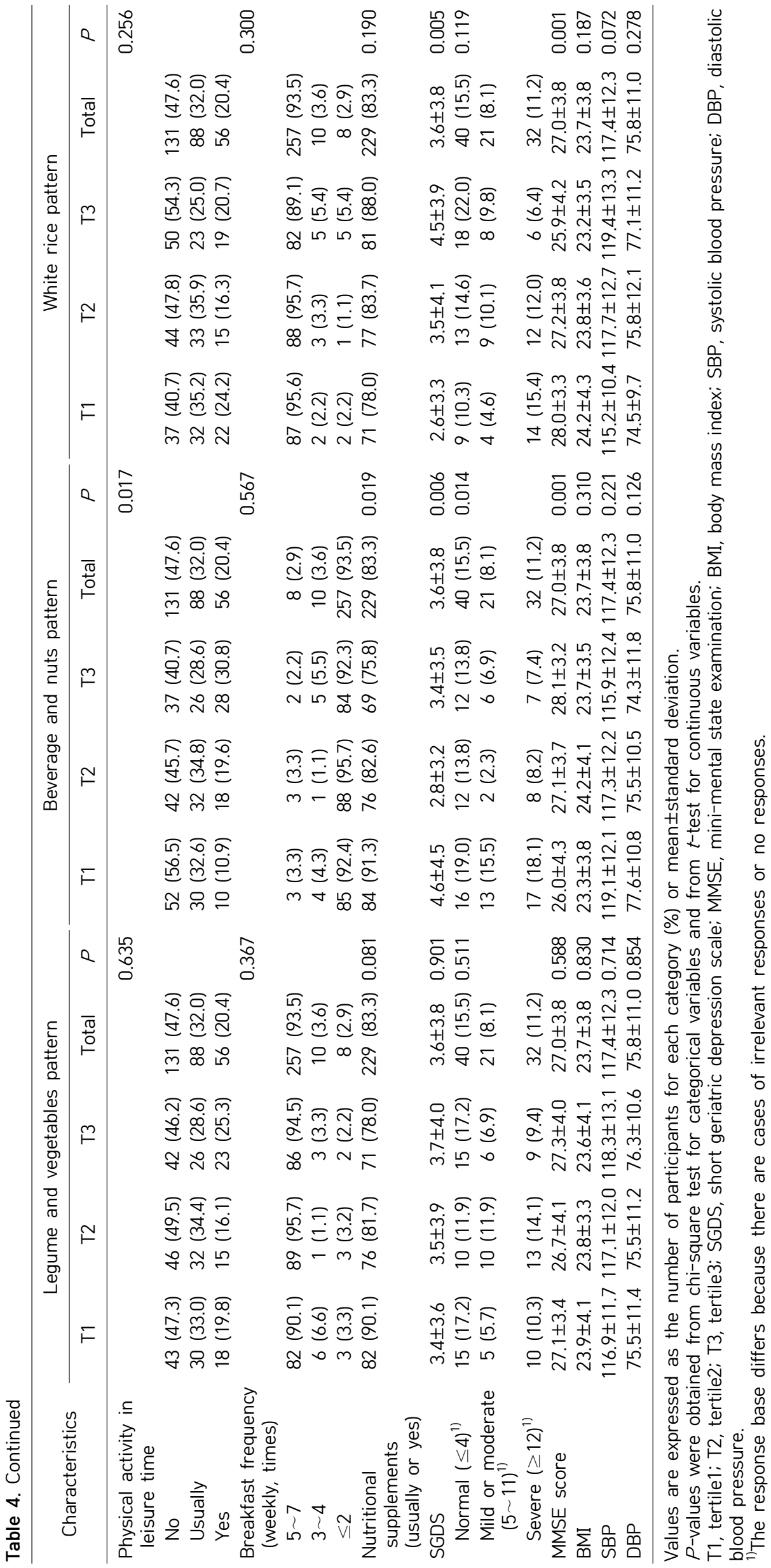




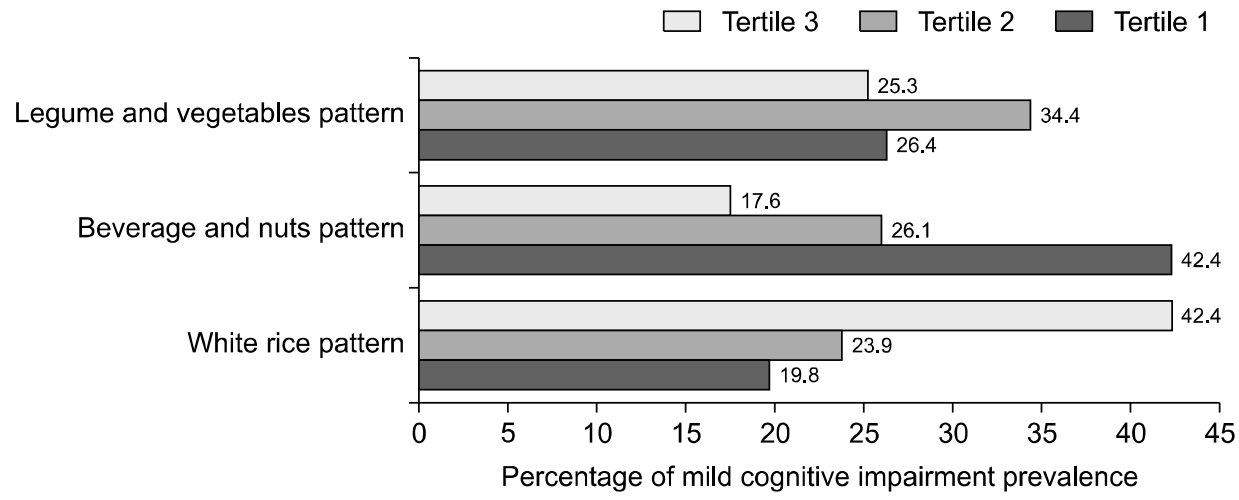

Fig. 2. Percentage of mild cognitive impairment prevalence across the tertiles of dietary pattern score. The prevalence of adult patients over aged 50 years with mild cognitive impairment decreased with an increase in the "beverage and nuts" pattern score, from the lowest tertile $(42.4 \%)$ to the highest tertile (17.6\%). However, as the white rice pattern score increased, the prevalence of mild cognitive impairment increased.
Intake frequency of food groups and food items according to the dietary pattern

Table 6 shows the intake level over the last year by food group as surveyed using the SQ-FFQ as the tertile of the dietary pattern score. In the legumes and vegetable pattern, the intake of white rice, rice-cake, soup and stew, legumes, eggs, red meat and processed meat, poultry, fish, vegetables, seaweed, fruit, dairy products, and nuts increased significantly in higher tertiles. In the "beverage and nuts" pattern, the intake of 20 food groups except for vegetables showed a significant difference according to the pattern score tertile. The subjects of the "beverage and nuts" pattern had a low level of vegetables intake, then the intake of salty vegetables was low according to tertile increased. Except for white rice, soup and stew, and legumes, the food group intake frequency was increased significantly between $\mathrm{T} 1$ to T3. This pattern was characterized by intake snacks such as drinks, nuts, sweets, fruits, rice cakes, coffee, tea, and bread, which resulted in a decrease of salty vegetables with high salt content as tertile increased. In the white rice pattern, the intake frequency of white rice, flour-based food, eggs, red meat and processed meat, poultry, and fish increased significantly with increasing tertile. On the other hand, the intake frequency of multigrain rice, fruit, and dairy products, decreased significantly as the tertile increased from $\mathrm{T} 1$ to $\mathrm{T} 3$.

\section{Adjusted odds ratio (AORs) for $\mathrm{MCI}$ by tertile of each dietary pattern score}

The AOR and the $95 \%$ CI for MCI by the tertile of each dietary pattern score are shown in Table 7. The "legumes and vegetable" pattern was not significantly associated with the risk of developing MCI in the crude or fully adjusted Model 1 (adjusted for sex, age, inhabitation, education, current disease, self-reported dental condition, sleep duration, alcohol consumption status, smoking status, physical activity in leisure time, nutritional supplements, and SGDS). The "beverage and nuts" pattern was associated with reduced odds of high MCI after fully adjusting for covariates (Model 1) [T3 vs. T1, AOR: 0.333; 95\% confidence interval $(\mathrm{CI}): 0.133 \sim 0.831 ; P<0.05]$.
The white rice pattern was associated with increased odds of high MCI in the crude analysis (T3 vs. T1, odd ratio: 2.984; 95\% CI: $1.541 \sim 5.780 ; P<0.01)$.

\section{DISCUSSION}

We identified the "legumes and vegetables", "beverage and nuts", and "white rice" patterns among participants aged $\geq 50$ years. The "beverage and nuts" pattern was negatively associated with the prevalence of high MCI, independent of education, self-reported dental condition, physical activity in leisure time, nutritional supplements, and SGDS. In contrast, the white rice pattern was positively associated with a risk of mild impairment, independent of sex, age, inhabitation, sleep duration, smoking status, and SGDS.

It has become increasingly important to consider the relevance of dietary patterns that reflect overall diet and dietary behavior to prevent and delay age-related cognitive decline. Previous systematic reviews and meta-analyses have shown the role of dietary patterns in cognitive function (Allès et al., 2012; Singh et al., 2014; van de Rest et al., 2015; Petersson and Philippou, 2016). Furthermore, Mediterranean diet (Valls-Pedret et al., 2015), Mediterranean-Dietary Approaches to Stop Hypertension (DASH) and Mediterranean-DASH Intervention for Neurodegenerative Delay diets (McEvoy et al., 2017) are associated with significantly better cognitive function and reduced risk of cognitive impairment. In the Melbourne Collaborative Cohort Study, fruit intake was positively associated with successful aging, including mental health and physical function, while meat/fat patterns were negatively associated (Hodge et al., 2014).

Based on these previous studies, the dietary patterns that affect cognitive function using multiple approaches were derived. In general, intake of more fruit, vegetables, fish, nuts, and higher fat dairy products have been found to have a beneficial effect on cognitive function.

The Whitehall II prospective cohort study evaluated that inflammatory diet patterns were associated with increased cognitive decline (Ozawa et al., 2017). Previous 


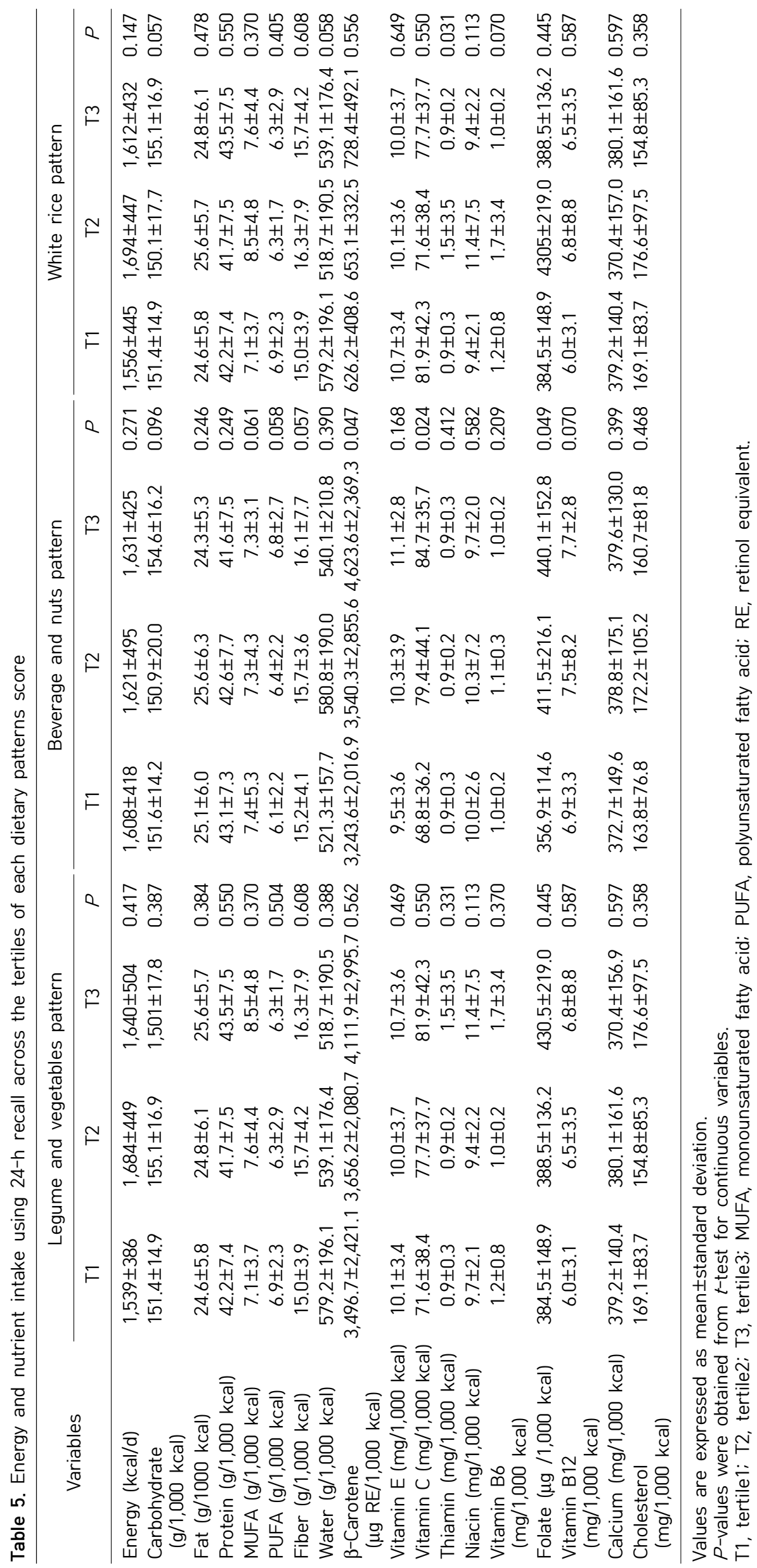




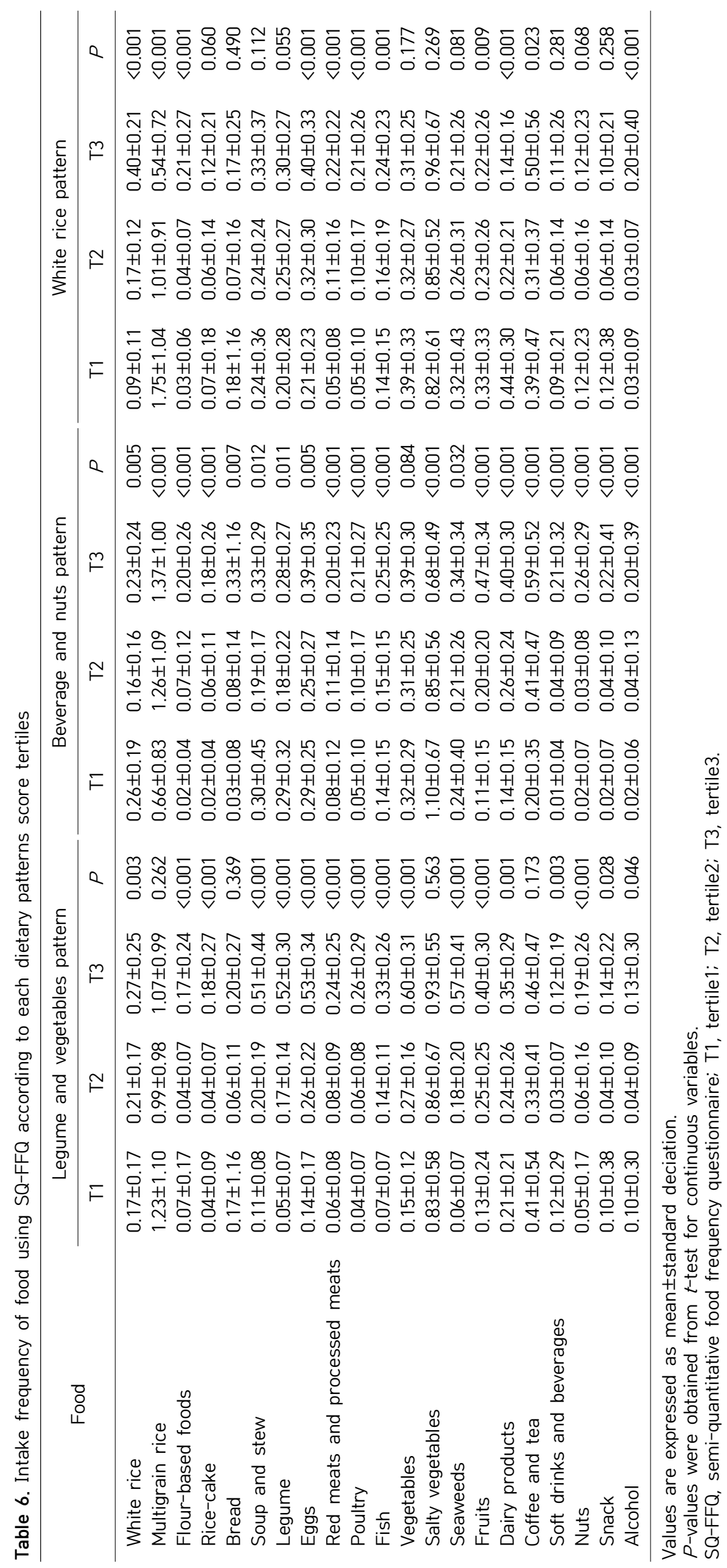


Table 7. Adjusted odds ratios (AOR) for mild cognitive impairment

\begin{tabular}{|c|c|c|c|c|c|}
\hline \multirow{2}{*}{ Dietary patterns } & \multirow{2}{*}{ MCI (n)/Total (n) } & \multicolumn{2}{|c|}{ Crude } & \multicolumn{2}{|c|}{ Model 1} \\
\hline & & $\mathrm{OR}^{1 / 2)}$ & $95 \% \mathrm{CI}^{1)}$ & $\mathrm{AOR}^{1 / 3)}$ & $95 \% \mathrm{CI}^{1)}$ \\
\hline \multicolumn{6}{|c|}{ Legume and vegetables pattern } \\
\hline Tertile1 & $24 / 91$ & Reference & & Reference & \\
\hline Tertile2 & $32 / 93$ & 1.464 & $0.778 \sim 2.757$ & 1.175 & $0.587 \sim 2.351$ \\
\hline Tertile3 & $23 / 91$ & 0.944 & $0.486 \sim 1.834$ & 0.949 & $0.466 \sim 1.935$ \\
\hline$P$ for trend & & 0.870 & & 0.931 & \\
\hline \multicolumn{6}{|c|}{ Beverage and nuts pattern } \\
\hline Tertile1 & $39 / 92$ & Reference & & Reference & \\
\hline Tertile2 & $24 / 92$ & $0.480^{*}$ & $0.257 \sim 0.894$ & 0.833 & $0.370 \sim 1.876$ \\
\hline Tertile3 & $16 / 91$ & $0.290^{* * *}$ & $0.147 \sim 0.572$ & $0.333 *$ & $0.133 \sim 0.831$ \\
\hline$P$ for trend & & $<0.001$ & & 0.014 & \\
\hline \multicolumn{6}{|l|}{ White rice pattern } \\
\hline Tertile1 & $18 / 91$ & Reference & & Reference & \\
\hline Tertile2 & $22 / 92$ & 1.275 & $0.630 \sim 2.577$ & 0.975 & $0.399 \sim 2.382$ \\
\hline Tertile3 & $39 / 92$ & $2.984^{* *}$ & $1.541 \sim 5.780$ & 1.876 & $0.750 \sim 4.690$ \\
\hline$P$ for trend & & 0.001 & & 0.094 & \\
\hline
\end{tabular}

1)Logistic regression analysis were used to estimate the $\mathrm{OR}$ and $95 \% \mathrm{CI}$ of $\mathrm{MCI}$ based on increasing the pattern score tertiles. ${ }^{2} \mathrm{OR}$ : without adjusting (crude).

${ }^{3)}$ AOR: adjusted for sex, age, inhabitation, education, self-reported dental condition, sleep duration, alcohol consumption status, smoking status, physical activity in leisure time, nutritional supplement, short geriatric depression scale (SGDS) (Model 1).

${ }^{*} P<0.05,{ }^{* *} P<0.01,{ }^{* * *} P<0.001$.

$\mathrm{MCI}$, mild cognitive impairment; $\mathrm{OR}$, odds ratio; $\mathrm{CI}$, confidence interval.

studies have assessed the role of these food groups on cognitive function (Gómez-Pinilla, 2008; Barbour et al., 2014; Solfrizzi et al., 2017). Flavonoid-rich fruits (Polidori et al., 2009) and nuts rich (in vitamins, minerals, MUFA, and PUFA (Barbour et al., 2014; Solfrizzi et al., 2017) that affect glucose metabolism, insulin resistance, and inflammatory mediators have been reported to have an impact on overall cognitive performance. Thus, the effect of fruit on cognitive function is not consistent. A study reported that fruit intake is associated with an increased risk of cognitive impairment due to high glycemic index and presence of simple sugars (Staubo et al., 2017).

In our study, "beverage and nuts" pattern, which is characterized by a high consumption of beverages, nuts, sweet foods, fruit, rice-cakes, coffee, tea, and bread, consisted of a combination of healthy and unhealthy food groups, such as in the study by Chan et al. (2013). At the current research level, it is not easy to interpret the relevance of our "beverage and nuts" pattern on cognitive function.

A recent systematic review of several cross-sectional studies and longitudinal population-based studies suggested that coffee and tea intake had a protective effect on cognitive impairment in older people. Although there were some limitations (such as using a dose-response analysis and cognitive domains), this review reported that this association was stronger in females than in males (Panza et al., 2015). Barbour et al. (2014) examined the effects of nut consumption on blood pressure, glucose regulation, endothelial vasodilator function, arterial compliance, inflammatory biomarkers, and cognitive function through several epidemiological or intervention studies. The effect of nut intake on cognitive function was found to be limited (Barbour et al., 2014). Therefore, we consider that more evidence from controlled intervention clinical trials is needed before determining whether nuts are beneficial. As is known, beverages such as soft drinks (cola, soda, and fruit juice soda), fruit juice, grain powder beverages, and rice beverages and snacks, such as cookies, crackers, chocolate, and ice cream, have a high sugar content. So far, there have been controversial results between studies on the effect that the consumption of beverages on cognitive function (Kakutani et al., 2019). An in-depth investigation is needed that takes into account different types of beverage.

As reviewed by Stephan et al. (2010) metabolic changes increase the risk of metabolic syndrome and may eventually increase cognitive decline (Yaffe et al., 2004). These results show that the positive effects of the "beverage and nuts" pattern on cognitive impairment might be due to the combined and synergistic results of various components, rather than simply a food component.

As shown Table 5, compared to the two other dietary patterns, the "beverage and nuts" pattern showed that $\beta$-carotene, vitamin $C$, and folate intake were significantly increased as the pattern score grows. The intake levels of $\beta$-carotene, vitamin $C$, and folate in the "beverage and nuts" pattern met the dietary intake levels recommended in the Dietary Reference Intakes for Koreans (Kim et al., 2015). A previous study reported that vitamin B and folic acid intake, which lowers plasma homocysteine levels, is associated with improved overall cognitive function and 
memory (Kim et al., 2014; Agnew-Blais et al., 2015). Staubo et al. (2017) confirmed that $\beta$-carotene intake is associated with cognitive function using dorsolateral, prefrontal, and temporal pole computed tomography. Li et al. (2012) also demonstrated that vitamin C and $\beta$-carotene have protective effects against the risk of Alzheimer's disease. However, since not all of the food consumed is absorbed into the body, further studies are warranted to explore the relationship between the intake of antioxidant nutrients (vitamin $C$ and $\beta$-carotene), which increases in concentration with the rising "beverage and nuts" pattern and plasma concentration.

In our study, the white rice pattern was found to be positively associated with increased cognitive decline in the crude analysis, but not after being fully adjusted. According to Korczak et al. (2016) inadequate intake of grain-based foods (namely, higher white rice intake and lower whole-grain intake) results in unbalanced mineral levels and insufficient intake of antioxidant nutrients, which is associated with an increased risk of developing MCI. It is believed that Koreans have unique dietary patterns, and the types of foods that make up the patterns are so diverse that it may be difficult to obtain consistent results on disease effects. We found no association between the legumes and vegetable pattern and cognitive impairment.

Our study has several limitations. First, we conducted a cross-sectional study that examined the dietary pattern and cognitive function for a specific period using a PCA analysis (a posteriori approach). In brief, cross-sectional studies cannot include all possible diet categories and cannot measure all aspects of the diet with absolute precision. We calibrated residual confounding factors in the analysis to minimize potential limitations. Second, our results cannot be generalized as our study participants were not a representative sample of Koreans over 50 years old. Despite the limitations stated, our study is meaningful as it is the first to analyze the relationship between cognitive status and dietary patterns of participants over 50 years old living in Gwangju province. Moreover, PCA analysis used in our study is widely used to derive dietary patterns in nutrition epidemiology, and our dietary patterns were similar to those obtained in previous studies that used a PCA analysis (Chan et al., 2013; Kim et al., 2015).

In conclusion, the present cross-sectional study identified three dietary patterns, "legumes and vegetables", "beverages and nuts", and "white rice". The "beverage and nuts" pattern, which is characterized by a high consumption of beverages, nuts, sweet foods, fruit, ricecakes, coffee, tea, and bread, was negatively associated with the prevalence of high MCI among Korean adults over 50 years old. In the future, longitudinal populationbased studies and randomized clinical trials are required to confirm the effect of potential dietary patterns on cognitive impairment and to reveal the underlying mechanism of their association.

\section{AUTHOR DISCLOSURE STATEMENT}

The authors declare no conflict of interest.

\section{REFERENCES}

Agnew-Blais JC, Wassertheil-Smoller S, Kang JH, Hogan PE, Coker LH, Snetselaar LG, et al. Folate, vitamin B-6, and vitamin B-12 intake and mild cognitive impairment and probable dementia in the Women's Health Initiative Memory Study. J Acad Nutr Diet. 2015. 115:231-241.

Albert MS, Blacker D. Mild cognitive impairment and dementia. Annu Rev Clin Psychol. 2006. 2:379-388.

Allès B, Samieri C, Féart C, Jutand MA, Laurin D, BarbergerGateau P. Dietary patterns: a novel approach to examine the link between nutrition and cognitive function in older individuals. Nutr Res Rev. 2012. 25:207-222.

Barbour JA, Howe PR, Buckley JD, Bryan J, Coates AM. Nut consumption for vascular health and cognitive function. Nutr Res Rev. 2014. 27:131-158.

Cederholm T, Salem N Jr, Palmblad J. $\omega$-3 fatty acids in the prevention of cognitive decline in humans. Adv Nutr. 2013. 4:672676.

Chan R, Chan D, Woo J. A cross sectional study to examine the association between dietary patterns and cognitive impairment in older Chinese people in Hong Kong. J Nutr Health Aging. 2013. 17:757-765.

Dong L, Xiao R, Cai C, Xu Z, Wang S, Pan L, et al. Diet, lifestyle and cognitive function in old Chinese adults. Arch Gerontol Geriatr. 2016. 63:36-42.

Eshkoor SA, Hamid TA, Mun CY, Ng CK. Mild cognitive impairment and its management in older people. Clin Interv Aging. 2015. 10:687-693.

Feskanich D, Rimm EB, Giovannucci EL, Colditz GA, Stampfer MJ, Litin LB, et al. Reproducibility and validity of food intake measurements from a semiquantitative food frequency questionnaire. J Am Diet Assoc. 1993. 93:790-796.

Freedman LS, Midthune D, Arab L, Prentice RL, Subar AF, Willett $\mathrm{W}$, et al. Combining a food frequency questionnaire with 24hour recalls to increase the precision of estimation of usual dietary intakes-evidence from the validation studies pooling project. Am J Epidemiol. 2018. 187:2227-2232.

Gómez-Pinilla F. Brain foods: the effects of nutrients on brain function. Nat Rev Neurosci. 2008. 9:568-578.

Greenberg SA. How to try this: the Geriatric Depression Scale: Short Form. Am J Nurs. 2007. 107:60-70.

Hodge AM, O’Dea K, English DR, Giles GG, Flicker L. Dietary patterns as predictors of successful ageing. J Nutr Health Aging. 2014. 18:221-227.

Hoffmann K, Schulze MB, Schienkiewitz A, Nöthlings U, Boeing $\mathrm{H}$. Application of a new statistical method to derive dietary patterns in nutritional epidemiology. Am J Epidemiol. 2004. 159:935-944.

Huang CQ, Dong BR, Wu HM, Zhang YL, Wu JH, Lu ZC, et al. Association of cognitive impairment with serum lipid/lipoprotein among Chinese nonagenarians and centenarians. Dement Geriatr Cogn Disord. 2009. 27:111-116.

Jang IM, Lee KB, Roh H, Ahn MY. Prevalence and risk factors of 
dementia and MCI in community-dwelling elderly Koreans. Dement Neurocogn Disord. 2014. 13:121-128.

Jiang X, Huang J, Song D, Deng R, Wei J, Zhang Z. Increased consumption of fruit and vegetables is related to a reduced risk of cognitive impairment and dementia: meta-analysis. Front Aging Neurosci. 2017. 9:18. https://doi.org/10.3389/fnagi.2017. 00018

Kakutani S, Watanabe H, Murayama N. Green tea intake and risks for dementia, Alzheimer's disease, mild cognitive impairment, and cognitive impairment: a systematic review. Nutrients. 2019. 11:1165. https://doi.org/10.3390/nu11051165

Kesse-Guyot E, Assmann KE, Andreeva VA, Ferry M, Hercberg S, Galan P; SU.VI.MAX 2 Research Group. Consumption of dairy products and cognitive functioning: findings from the SU.VI. MAX 2 study. J Nutr Health Aging. 2016. 20:128-137.

Khosravi M, Sotoudeh G, Majdzadeh R, Nejati S, Darabi S, Raisi $\mathrm{F}$, et al. Healthy and unhealthy dietary patterns are related to depression: a case-control study. Psychiatry Investig. 2015. 12: 434-442.

Kim H, Kim G, Jang W, Kim SY, Chang N. Association between intake of B vitamins and cognitive function in elderly Koreans with cognitive impairment. Nutr J. 2014. 13:118. https://doi. org/10.1186/1475-2891-13-118

Kim J, Yu A, Choi BY, Nam JH, Kim MK, Oh DH, et al. Dietary patterns and cognitive function in Korean older adults. Eur J Nutr. 2015. 54:309-318.

Kline P. An easy guide to factor analysis. 1st ed. Routledge, London, UK. 1994. p 14-27.

Korczak R, Jones JM, Peña RJ, Braun HJ. CIMMYT series on carbohydrates, wheat, grains, and health: carbohydrates and their grain sources: a review on their relationships to brain health. Cereal Foods World. 2016. 61:143-156.

Lee KW, Cho MS. The traditional Korean dietary pattern is associated with decreased risk of metabolic syndrome: findings from the Korean National Health and Nutrition Examination Survey, 1998-2009. J Med Food. 2014. 17:43-56.

Li FJ, Shen L, Ji HF. Dietary intakes of vitamin E, vitamin C, and $\beta$-carotene and risk of Alzheimer's disease: a meta-analysis. J Alzheimers Dis. 2012. 31:253-258.

McCann SE, Marshall JR, Brasure JR, Graham S, Freudenheim JL. Analysis of patterns of food intake in nutritional epidemiology: food classification in principal components analysis and the subsequent impact on estimates for endometrial cancer. Public Health Nutr. 2001. 4:989-997.

McEvoy CT, Guyer H, Langa KM, Yaffe K. Neuroprotective diets are associated with better cognitive function: the health and retirement study. J Am Geriatr Soc. 2017. 65:1857-1862.

Ogata S, Tanaka H, Omura K, Honda C, Osaka Twin Research Group, Hayakawa K. Association between intake of dairy products and short-term memory with and without adjustment for genetic and family environmental factors: A twin study. Clin Nutr. 2016. 35:507-513.

Osler M, Helms Andreasen A, Heitmann B, Høidrup S, Gerdes U, Mørch Jørgensen L, et al. Food intake patterns and risk of coronary heart disease: a prospective cohort study examining the use of traditional scoring techniques. Eur J Clin Nutr. 2002. 56:568-574.

Ozawa M, Ninomiya T, Ohara T, Doi Y, Uchida K, Shirota T, et al. Dietary patterns and risk of dementia in an elderly Japanese population: the Hisayama Study. Am J Clin Nutr. 2013. 97: 1076-1082.

Ozawa M, Shipley M, Kivimaki M, Singh-Manoux A, Brunner EJ. Dietary pattern, inflammation and cognitive decline: the
Whitehall II prospective cohort study. Clin Nutr. 2017. 36:506512.

Panza F, Solfrizzi V, Barulli MR, Bonfiglio C, Guerra V, Osella A, et al. Coffee, tea, and caffeine consumption and prevention of late-life cognitive decline and dementia: a systematic review. J Nutr Health Aging. 2015. 19:313-328.

Petersson SD, Philippou E. Mediterranean diet, cognitive function, and dementia: a systematic review of the evidence. Adv Nutr. 2016. 7:889-904.

Polidori MC, Praticó D, Mangialasche F, Mariani E, Aust O, Anlasik T, et al. High fruit and vegetable intake is positively correlated with antioxidant status and cognitive performance in healthy subjects. J Alzheimers Dis. 2009. 17:921-927.

Schulze MB, Hoffmann K, Kroke A, Boeing H. An approach to construct simplified measures of dietary patterns from exploratory factor analysis. Br J Nutr. 2003. 89:409-419.

Sheikh JI, Yesavage JA. Geriatric depression scale (GDS): recent evidence and development of a shorter version. In: Brink TL, editor. Clinical Gerontology: A Guide to Assessment and Intervention. The Haworth Press, Inc., New York, NY, USA. 1986. p 165-174.

Shin D, Lee KW, Kim MH, Kim HJ, An YS, Chung HK. Identifying dietary patterns associated with mild cognitive impairment in older Korean adults using reduced rank regression. Int J Environ Res Public Health. 2018. 15:100. https://doi.org/10. 3390/ijerph15010100

Singh B, Parsaik AK, Mielke MM, Erwin PJ, Knopman DS, Petersen RC, et al. Association of Mediterranean diet with mild cognitive impairment and Alzheimer's disease: a systematic review and meta-analysis. J Alzheimers Dis. 2014. 39:271-282.

Smith PJ, Blumenthal JA. Dietary factors and cognitive decline. J Prev Alzheimers Dis. 2016. 3:53-64.

Solfrizzi V, Custodero C, Lozupone M, Imbimbo BP, Valiani V, Agosti P, et al. Relationships of dietary patterns, foods, and micro- and macronutrients with Alzheimer's disease and latelife cognitive disorders: a systematic review. J Alzheimers Dis. 2017. 59:815-849.

Staubo SC, Aakre JA, Vemuri P, Syrjanen JA, Mielke MM, Geda YE, et al. Mediterranean diet, micronutrients and macronutrients, and MRI measures of cortical thickness. Alzheimers Dement. 2017. 13:168-177.

Stephan BCM, Wells JCK, Brayne C, Albanese E, Siervo M. Increased fructose intake as a risk factor for dementia. J Gerontol A Biol Sci Med Sci. 2010. 65:809-814.

Tombaugh TN, McIntyre NJ. The mini-mental state examination: a comprehensive review. J Am Geriatr Soc. 1992. 40:922-935.

Valls-Pedret C, Sala-Vila A, Serra-Mir M, Corella D, de la Torre R, Martínez-González MÁ, et al. Mediterranean diet and agerelated cognitive decline: a randomized clinical trial. JAMA Intern Med. 2015. 175:1094-1103.

van de Rest O, Berendsen AA, Haveman-Nies A, de Groot LC. Dietary patterns, cognitive decline, and dementia: a systematic review. Adv Nutr. 2015. 6:154-168.

Wimo A, Jönsson L, Bond J, Prince M, Winblad B; Alzheimer Disease International. The worldwide economic impact of dementia 2010. Alzheimers Dement. 2013. 9:1-11.e3.

Xu W, Wang H, Wan Y, Tan C, Li J, Tan L, et al. Alcohol consumption and dementia risk: a dose-response meta-analysis of prospective studies. Eur J Epidemiol. 2017. 32:31-42.

Yaffe K, Kanaya A, Lindquist K, Simonsick EM, Harris T, Shorr $\mathrm{RI}$, et al. The metabolic syndrome, inflammation, and risk of cognitive decline. JAMA. 2004. 292:2237-2242. 